\title{
Moyens d'estimer \\ la valeur économique des installations de transiert d'énergie par la simulation mathématique d'exploitation. Application de ces données à l'aménagèement et au choix des sites
}

\author{
par P. Gérard \\ Chef de la Division Projets-Prospection au D.E.P.H., \\ E.D.F., Paris
}

\section{Généralités}

Pour estimer la valeur de ce moyen qu'on appelle schématiquement «le pompage», on va comme d'habitude comparer son coût au bénéfice qu'il apporte au système de production.

La valeur de l'hydraulique classique était, à E.D.F., estimée par la fameuse Note bleue qui fut sans doute, à l'échelle peut-être mondiale, un des bons exemples d'analyse rationnelle des choix budgétaires. On définissait la valeur du service rendu par le projet hydraulique que nous offre 1a nature, par référence au thermique de main d'homme qu'on aurait toujours pu construire à la place : la rigueur de la comparaison où rien n'était laissé dans l'ombre faisait la valeur de cette méthode, qui mettait aussi en évidence l'importance fondamentale du choix politique du taux d'actualisation. Ces principes généraux restent toujours valables, ainsi que la Note bleue dans son principe.

Mais, pour que cette méthode s'applique bien telle quelle, il faut que le projet et son étalon de comparaison n'influent pas l'un sur l'autre. En outre, la méthode permet surtout de classer les projets, et d'optimiser leurs dimensions. Elle dit si les projets sont acceptables ou non, mais elle ne dit pas combien de projets acceptables il faut réaliser: il reste à faire un ajustement entre la demande et la quantité de bons projets à réaliser. En pratique, on établit un échéancier avec le souci de répartir la charge de travail entre les diverses branches d'industrie.

Depuis que les moyens de main d'homme se sont très diversifiés (nucléaires de divers types, centrales au fuel de divers rendements, turbines à gaz, etc.), on ne sait plus bien quel est le moyen qui doit servir d'étalon à l'autre. On est ainsi amené à poser le problème en terme global : quel est le meilleur assemblage de tous les moyens de production possibles pour faire face à une demande elle-même constituée d'un assemblage de besoins de natures diverses. Les nouveaux outils de calculs permettent de résoudre globalement ce problème et d'arriver à une optimisation absolue par une méthode de retouches successives et de substitutions marginales. Par exemple, partant d'un parc donné et optimisé comprenant ou non déjà du pompage, on introduit dans ce parc un nouveau projet de pompage: la réoptimi- sation du parc conduira à des retouches, par exemple, à enlever de la turbine à gaz à laquelle le nouveau pompage se substitue partiellement, éventuellement à rajouter un peu de thermique de base qui fournit l'énergie d'heures creuses, à changer les durées de fonctionnement d'un grand nombre de moyens, tout ceci pour satisfaire la demande au coût minimum, c'est-à-dire pour la valeur minimum de la somme des annuités d'investissement, des charges fixes, des consommations et d'une estimation chiffrée du préjudice de défaillance.

Cette méthode globale est merveilleuse, mais sa perfection comporte un revers: c'est qu'on ne comprend plus bien ce qui se passe.

Avant de présenter les résultats globaux résultant de ce type d'étude, nous allons donc, par des approches partielles et un peu simplistes, essayer de montrer ce qui se passe, c'est-à-dire quel est le jeu de quelques paramètres principaux. Indépendamment des vérifications globales faites par l'ordinateur, ce type de démarche est nécessaire pour le projeteur qui doit comprendre ce qu'il doit rechercher et imaginer.

\section{Pompage et turbine à gaz}

La première approche simple consiste à remarquer que le pompage est essentiellement un moyen de pointe, et, par analogie avec le raisonnement Note bleue, à le comparer à l'étalon turbine à gaz, moyen de pointe qu'on pourrait toujours construire à la place.

Ce n'est déjà pas simple, mais on va voir cependant que l'essentiel des considérations économiques se déduit de cette comparaison.

Sur la base d'unité de 50 ou $60 \mathrm{GW}$, on admet que la turbine à gaz à un coût au $\mathrm{kW}$ installé de $500 \mathrm{~F}$, des charges fixes de $200 \mathrm{~F} / \mathrm{kW}$ et produit des $\mathrm{kWh}$ au coût proportionnel d'environ 4 à $5 \mathrm{c} / \mathrm{kWh}$.

L'étude des sites de pompage permet aussi (et c'est un avantage par rapport à l'hydraulique classique) d'annoncer certains coûts qui varient dans d'assez faibles limites: le matériel coûte toujours entre 200 et $250 \mathrm{~F} / \mathrm{kW}$, le génie 


\section{P. GERARD}

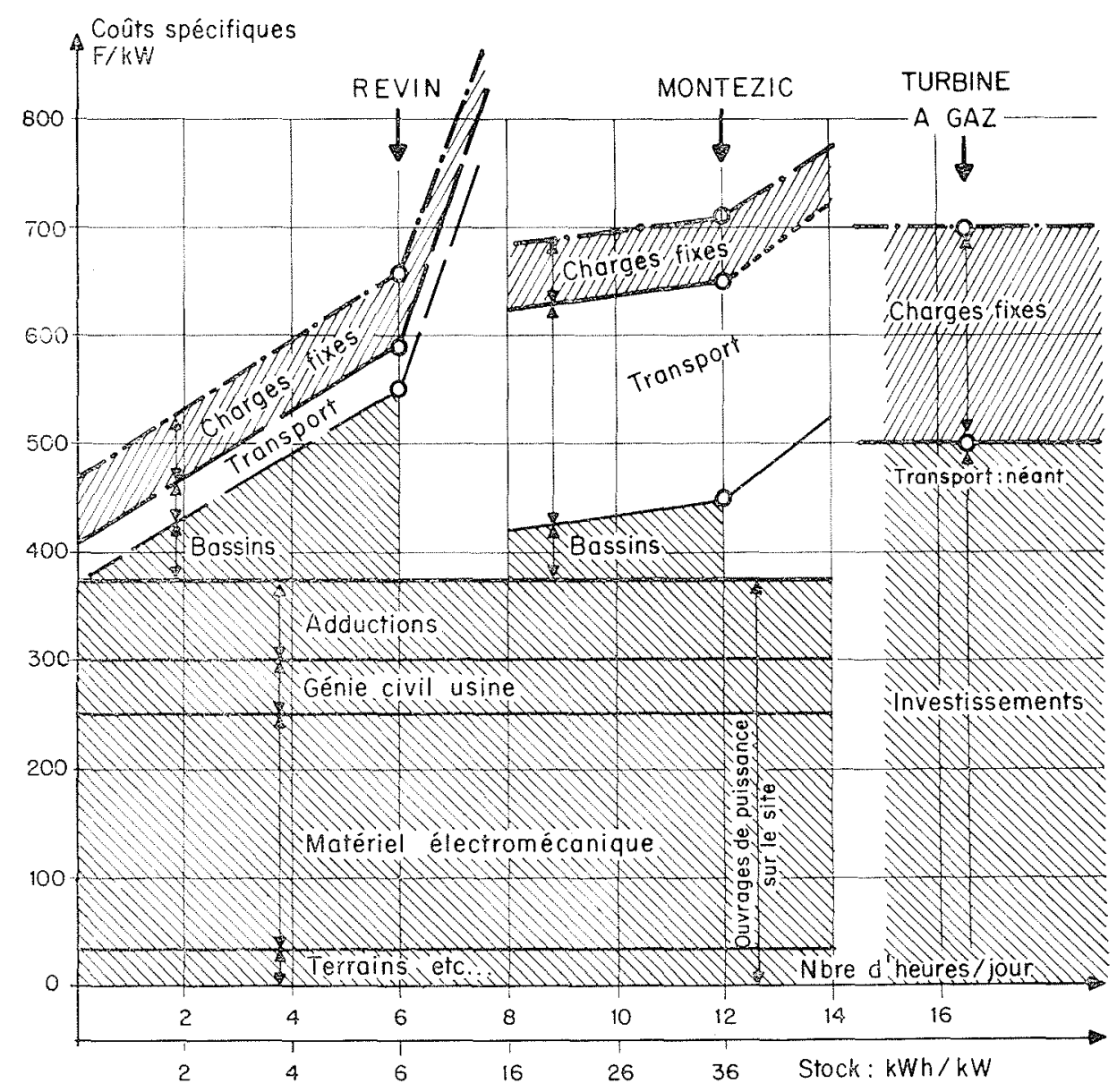

1/ Analyse des coûts de stations de pompage-types.

civil de l'usine (compris poste, accès, etc.) entre 40 et $60 \mathrm{~F} / \mathrm{kW}$, et les adductions entre 70 et $150 \mathrm{~F} / \mathrm{kW}$ (car le projeteur élimine dans sa prospection les mauvais sites où le rapport entre la distance horizontale entre bassins et la hauteur de chute est supérieur à 4 pour les hautes chutes, et 6 pour les basses chutes). On aboutit ainsi à un coût global sans les bassins de l'ordre de 300 à $400 \mathrm{~F} / \mathrm{kW}$ (tous frais inclus).

Mais le coût des bassins, lui, est très variable d'un site à l'autre. Exprimé en $\mathrm{kWh}$ stocké (ce qui est le paramètre significatif), il varie de zéro si les lacs existent, à près de $50 \mathrm{~F} / \mathrm{kWh}$ pour des cuvettes entièrement artificielles et des hauteurs de chute assez faibles.

Quant au coût proportionnel du kWh de pointe produit, il est égal à 1,4 fois (rendement du cycle) le coût proportionnel $\mathrm{du} \mathrm{kWh}$ acheté pour le pompage. Celui-ci est produit par les bonnes centrales au fuel ( $2 \mathrm{c} / \mathrm{kWh}$ environ) tant que le nucléaire n'est pas disponible en heures creuses, c'est-à-dire pas avant 1990. D'où un coût de production d'environ $2,8 \mathrm{c}$. On gagne ainsi de l'ordre de $1,5 \mathrm{c} / \mathrm{kWh}$ par rapport à la turbine à gaz: cela est bien clair que le pompage est d'autant plus compétitif avec la turbine à gaz qu'il s'agit de fournir beaucoup de $\mathrm{kWh}$ dans l'année tout en restant dans le domaine de la pointe, tandis que la turbine à gaz est d'autant plus intéressante qu'elle tourne peul.

Fournir beaucoup de $\mathrm{kWh}$, cela signifie tourner de nombreux jours par an et longtemps chaque jour. Or, tourner longtemps chaque jour, implique qu'on dispose d'un grand stockage de $\mathrm{kWh}$

On en revient ainsi au coût du stockage et on met en évidence le paramètre fondamental de la durée quotidienne de la fourniture. S'il s'agissait de fournir la pleine puissance seulement 3 ou $4 \mathrm{~h}$ par jour, même avec un coût de stockage cher, la station de pompage coûterait déjà moins que la turbine à gaz en investissements seuls. Abstraction faite de la nécessité de trouver de l'énergie d'heure creuse (mais il en faudrait alors peu), on ne construirait alors pratiquement pas de turbines à gaz. Mais il s'agit de fournir, chaque jour, plus longtemps et de stocker de quoi produire 6,8 ou $10 \mathrm{~h}$ par jour. A bassins identiques, le coût du $\mathrm{kWh}$ stocké est inversement proportionnel à la chute. Donc, les sites à stockage bon marché sont les sites de montagne, où, de plus, les cuvettes, qui peuvent être créées par des barrages sont économiques. Or, les montagnes, en France, sont loin de la région parisienne et du nord de la France qui constituent, jusqu'en 1985 ou 1990 , les seuls centres où on a vraiment besoin de pointe, précisément parce qu'il n'y a pas d'hydraulique. La turbine à gaz, par contre, peut s'installer près des besoins.

D'où lié au coût du stockage, un nouveau paramètre essentiel: la localisation qu'on introduira sous la forme d'une charge de transport.

On peut, tout de même, poursuivre la comparaison directe turbine à gaz-pompage en notant que la géographie de la France conduit, pour le pompage, à une certaine compen- 
I - Différence (Pompage - thermique C) exprimée en \% de l'opération la meilleure

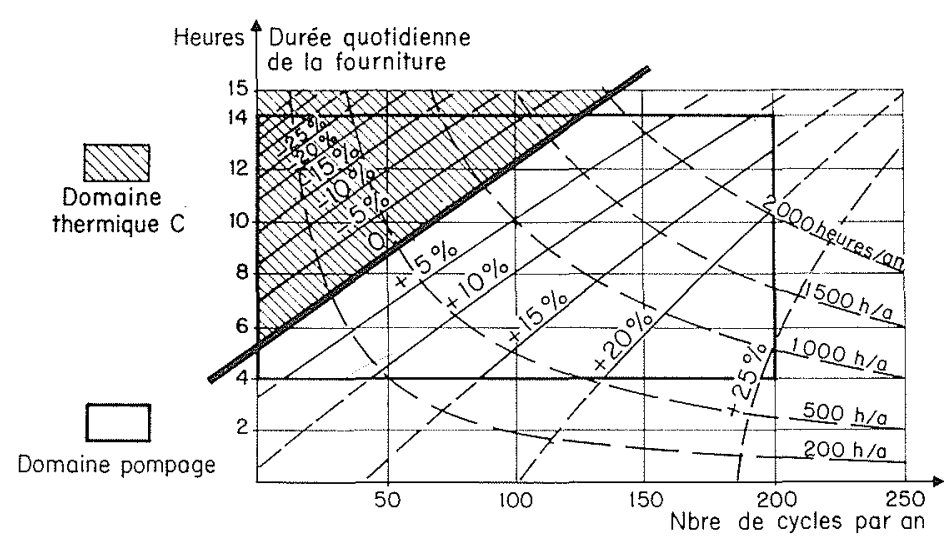

I-Différence (Pompage - thermique C) exprimée en froncs por $\mathrm{kW}$

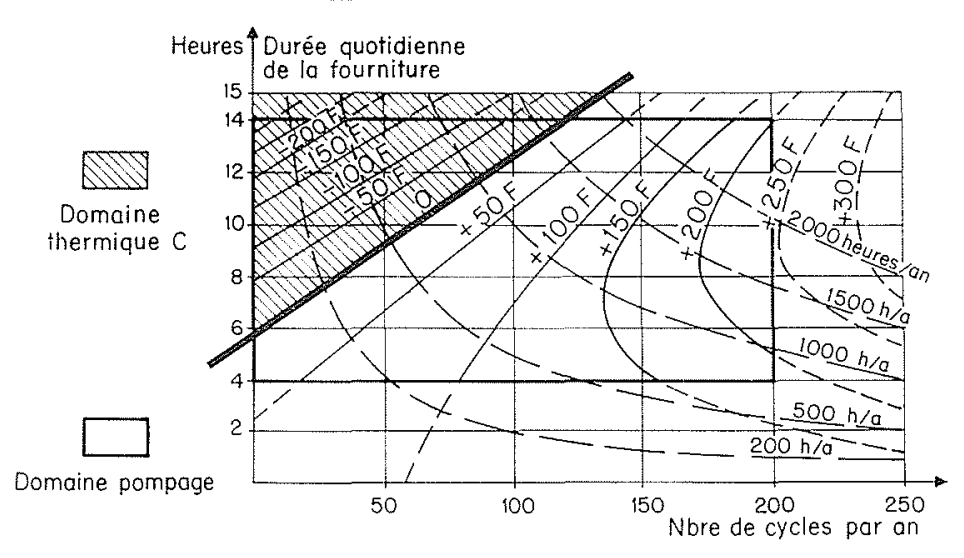

2/Comparaison schématique pompage-turbine ì gaz (thermique $\mathrm{C}$ ).

sation entre le coût du stockage et la charge de transport. $\mathrm{Si}$ le site est dans les Alpes, on stocke le $\mathrm{kWh}$ pour moins de $10 \mathrm{~F}$. Si on fournit $6 \mathrm{~h}$ (ce qui correspond à $8 \mathrm{~h}$ de pompage avec des machines de même puissance en turbine et en pompe, ce qui esi, en général, l'optimum), l'incidence sur le coût du stockage de la station est ainsi inférieure à $60 \mathrm{~F} / \mathrm{kW}$, mais il faut ajouter - actuellement - de 150 à $200 \mathrm{~F} / \mathrm{kW}$ de charges de transport; si, par contre, le site est près de Paris, on stocke le $\mathrm{kWh}$ pour plus de $30 \mathrm{~F}$, soit $180 \mathrm{~F} / \mathrm{kW}$ pour ces mêmes $6 \mathrm{~h}$, mais il $\mathrm{n}^{\prime} \mathrm{y}$ a que 20 ou $50 \mathrm{~F} / \mathrm{kW}$ de transport. Coût du stockage et charges de transport ont donc tendance à se compenser:

$$
60 F+200 F \simeq 180 F+50 F
$$

Le graphique $l$ résume le jeu de ces paramètres.

Finalement, la station de pompage, charges d'exploitation et de transport inclus livre sa puissance aux environs de Paris, au prix de 6 à $700 \mathrm{~F} / \mathrm{kW}$, pour $6 \mathrm{~h}$ de fourniture quotidienne. Si on se souvient que la turbine à gaz coûte $700 \mathrm{~F} / \mathrm{kW}$, on voit que le pompage, moins cher en coût proportionnel, est plutôt meilleur que la turbine à gaz pour cette fourniture de $6 \mathrm{~h}$.

Mais, si le réseau demande plus de $6 \mathrm{~h}$ par jour, la station de pompage doit recourir au report de fin de semaine pour prolonger sa fourniture quotidienne et rendre un service compétitif avec celui de la turbine à gaz. Or, l'eau du report hebdomadaire sert cinq fois moins souvent que l'eau utilisée quotidiennement : pour passer de $6 \mathrm{~h}$ de fourniture par jour à $10 \mathrm{~h}$, par exemple, le réservoir doit passer du volume 6 au volume $6+5$ fois $(10-6)=26$.

Les stations à longue durée de fourniture, c'est-à-dire à report de fin de semaine sont donc nécessairement situées en montagne, là où le stockage est bon marché. Le coûttype de ce stockage hebdomadaire est de 2 à $4 \mathrm{~F} / \mathrm{kWh}$, ce qui représente de 50 à $100 \mathrm{~F} / \mathrm{kW}$ pour $10 \mathrm{~h}$ de fourniture. Livrée dans la région parisienne, cette puissance de 10 ou $12 \mathrm{~h}$ risque alors d'être un peu plus chère au $\mathrm{kW}$ que celle de la turbine à gaz, mais c'est alors la grande quantité de $\mathrm{kWh}$ fournis à un coût proportionnel moindre que par la turbine à gaz qui donne sa valeur au projet de montagne.

Le diagramme 2 résume ceci et distingue nettement deux zones économiques où le pompage et la turbine à gaz sont, chacun dans leur domaine, le moyen le plus économique:

- le pompage s'il s'agit de fourniture de courte durée (4 h par jour par exemple), ou bien d'assez longue durée, mais de nombreux jours de l'année (200 fois $10 \mathrm{~h}$ par exemple);

- la turbine à gaz s'il s'agit d'une fourniture de longue durée, mais rarement dans l'année (30 fois $12 \mathrm{~h}$ par exemple).

On voit déjà que les deux moyens sont complémentaires et il serait bien étonnant qu'un modèle ne donne pas, à l'optimum, un mélange des deux procédés. 


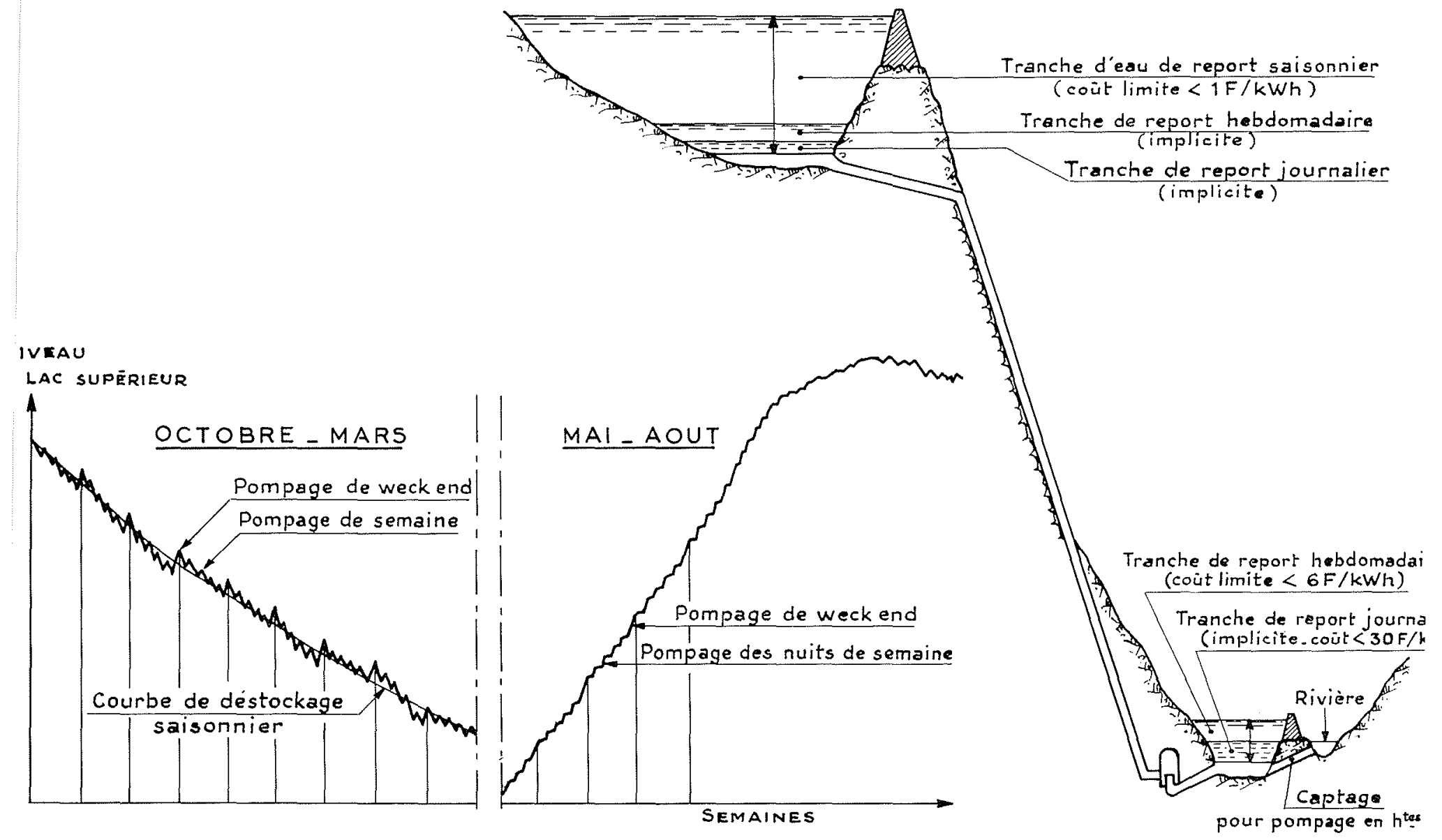

3/Schéma d'une installation de "pompage saisonnier ».

\section{Importance de la dispersion et des aléas}

Fournir avec du pompage une longue durée de fourniture, peu de jours dans l'année, cela pourrait, a priori, se faire avec un réservoir saisonnier. Ainsi, la turbine à gaz n'auraitelle plus aucun domaine économique.

En fait, il n'en est rien, car ce dont le système a besoin, ce n'est pas d'une longue fourniture régulièrement quelques jours chaque année, mais d'un petit nombre de jours en espérance mathématique. Certaines années difficiles, il faut le soutien d'une longue fourniture de nombreux jours, et dans la plupart des autres années, on n'a besoin de rien du tout. Ce n'est donc pas un petit lac saisonnier servant tous les ans, avec une forte puissance associée (ou un suréquipement des installations de lac existantes) qui résout le problème: ce serait un grand lac ne servant qu'une année sur cinq, ce qui est, bien sûr, économiquement impensable.

C'est donc, en fait, la variation des besoins et des aléas d'une année à l'autre qui fait l'intérêt spécifique de la turbine à gaz.

Les aléas d'une heure à l'autre, statistiquement voisins d'un jour à l'autre relèvent du pompage journalier qui, s'il est implanté assez près des grands centres de consommation, joue très bien le rôle de réserve tournante et de réglage fréquence-puissance. C'est le cas de Revin et de la plupart des stations de pompage étrangères implantées en général en zone thermique, en Allemagne notamment. On ne cherche pas, dans ce cas, à transférer le maximum d'énergie des heures creuses sur les heures pleines: on estime gagner plus en se servant du pompage pour sa souplesse et sa disponibilité. On ne produira, par exemple, que 0,6 ou $0,8 \mathrm{TWh}$ par GW.

Les aléas d'un jour à l'autre, statistiquement voisins d'une semaine à l'autre relèvent du pompage hebdomadaire qui permet, un jour, de fonctionner $8 \mathrm{~h}$, et le lendemain $13 \mathrm{~h}$, dans la limite des quelque 50 à $60 \mathrm{~h}$ fournies par les heures creuses de pompage en incluant celles de fin de semaine. On réalise, ce faisant, beaucoup de transfert d'énergie, de façon assez systématique. On produit, par exemple, de 1,5 à 2 TWh par GW.

Les aléas d'une semaine à l'autre, statistiquement voisins d'une année à l'autre, relèvent encore du pompage, mais du pompage saisonnier (schéma 3), qui est une variante de l'énergie de lac classique, où le stock est constitué l'été par du pompage à partir du niveau inférieur, depuis la vallée, au lieu d'être accumulé gravitairement au niveau supérieur par des adductions.

Mais les aléas d'une année sur l'autre, eux, ne relèvent plus d'aucun procédé hydraulique, mais de la turbine à gaz. Plus exactement, on trouve que l'optimum consiste à définir un programme de moyens lourds (dont le pompage fait partie) calé sur les prévisions de l'année moyenne et qui ne couvre donc pas toutes les éventualités, et de prévoir une petite marge supplémentaire (qui apparaît être de l'ordre de 

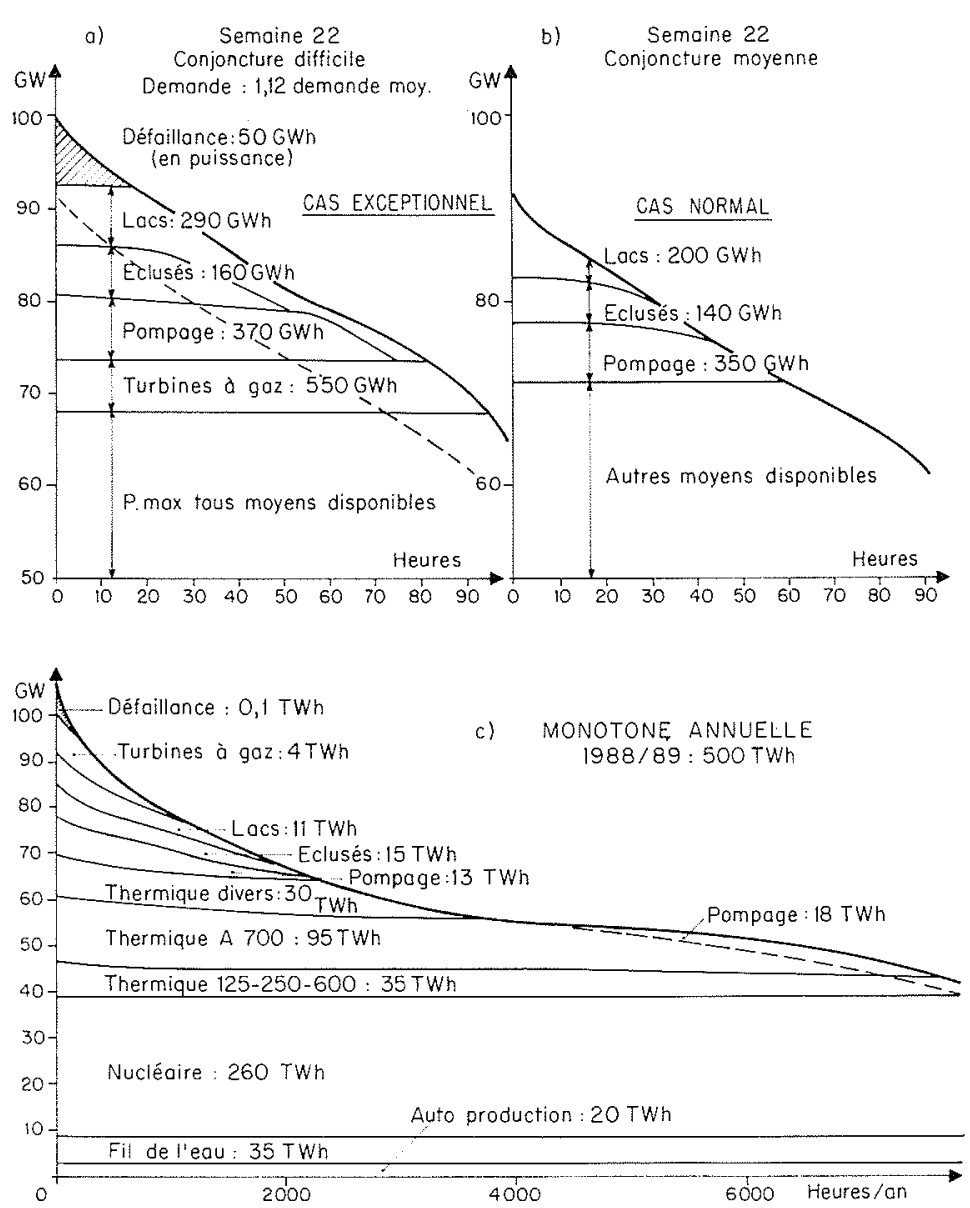

4/Comment se place la turbine ì gaz sur les monotones hebdomadaires et sur la monotone annuelle.

3 à $5 \%$ de la puissance installée) constituée de turbines à gaz, qui ont un rôle de réserve, pour satisfaire, le cas échéant, une demande supérieure à celle de la prévision la plus probable. En espérance mathématique, la turbine à gaz se place donc au-dessus du pompage sur la monotone annuelle moyenne, mais, en fait, sur les monotones hebdomadaires, ou bien elle se situe en dessous, ou bien elle est inexistante (graphique 4).

Précisément, la turbine à gaz est l'outil de production qui se construit le plus vite: elle peut être en service deux ans après la décision, contre quatre ans pour le pompage, cinq à six ans pour le thermique lourd ou l'hydraulique classique. C'est donc le moyen d'ajustement fin des programmes à faire intervenir au dernier moment si le programme lourd décidé à l'année $n-5$, s'avère un peu court à l'année $n-2$.

Ces considérations montrent l'importance, dans la définition des moyens de pointe, de passer en revue toute la gamme des situations aléatoires possibles des années à venir : c'est l'objet des modèles de simulation d'exploitation dont le rôle essentiel est de traduire en espérance mathématique l'intégration d'une multitude d'éventualités. On retiendra incidemment que la définition des moyens lourds relève d'une étude prévisionnelle à cinq ou six ans (ce qui se traduit par un écart-type des familles de demandes possibles d'environ $5 \%$ ), et la définition des moyens légers d'une étude à deux ans (ce qui se traduit par un écart-type d'environ $3 \%$ ). D'où la nécessité de modèles statistiques.

\section{Modèle de simulation d'exploitation}

On parlera ici du modèle GRETA (Gestion du réservoir avec thermique aléatoire) mis au point par le service EDF des Etudes et Recherches.

Son pas d'étude est la semaine. Pour le pompage, il s'adapte donc bien à l'étude du report hebdomadaire (ou saisonnier), mais il masque évidemment l'intérêt de l'adaptation fine du pompage à la demande, heure par heure, notion qui ne peut être mise en évidence que par un autre modèle à construire.

Il étudie quinze séquences d'hydraulicité, elles-mêmes associées, chaque semaine, à soixante conjonctures de demande et d'aléas thermiques, c'est-à-dire, qu'il optimise la gestion de neuf cents situations hebdomadaires, multipliées par cinquante-deux semaines pour donner les résultats globaux en espérance mathématique sur l'année.

Il travaille sur des monotones hebdomadaires de demande et empile au mieux quelque dix-sept types de thermique définis par leurs coûts proportionnels, après avoir tiré au sort leurs pannes, après avoir tenu compte de l'entretien programmé, après avoir placé le fil de l'eau, les éclusées, et calculé par un programme spécial déterminant la valeur de l'eau chaque semaine, la quantité optimale à déstocker du lac France. 


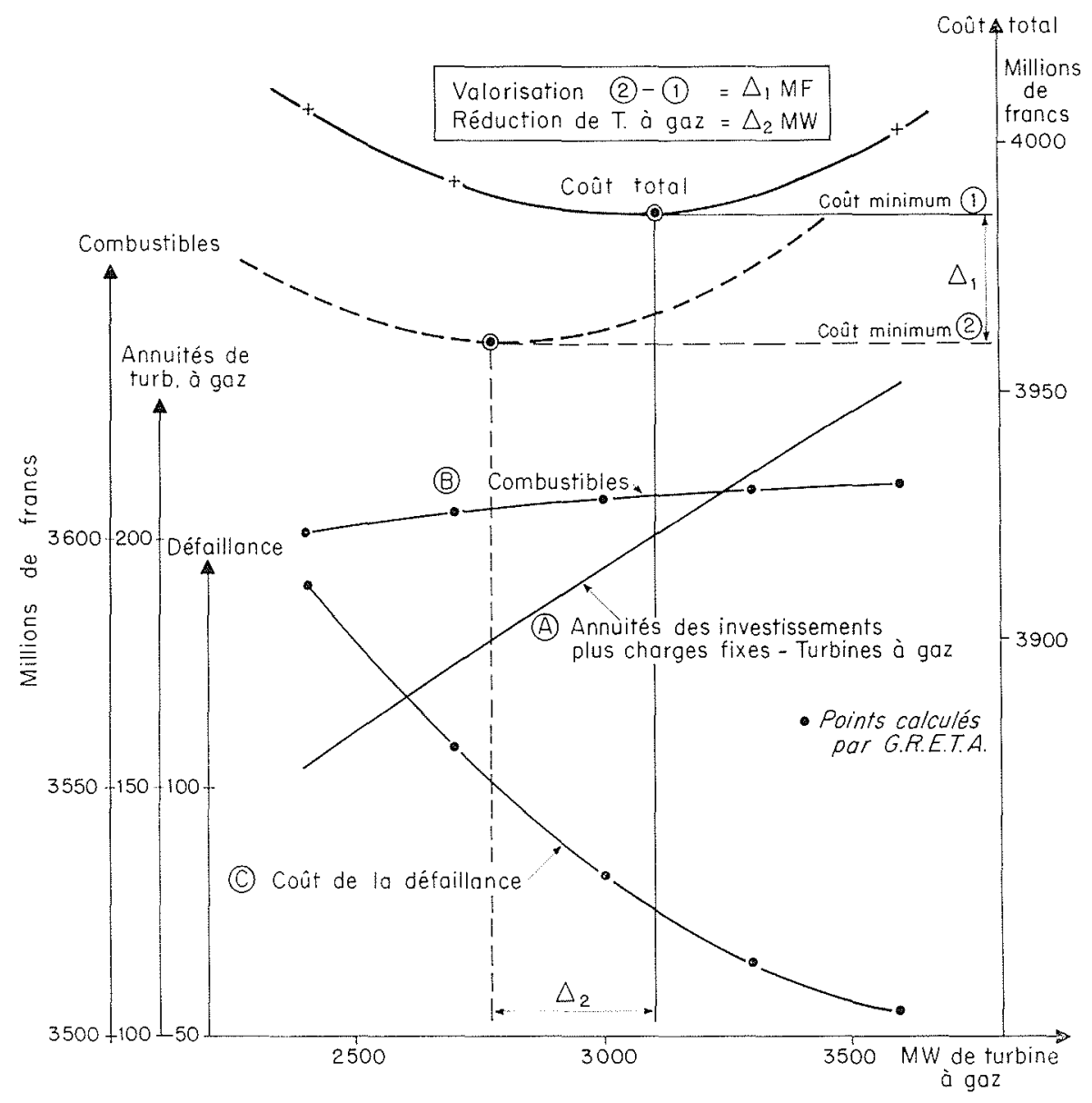

5/Optimisation du parc dans deux hypothèses de pompage.

Depuis deux ans, ce programme est couplé à un sousprogramme pompage qui recherche, chaque semaine, l'énergie économiquement productible pour le pompage, calcule son coût, la transforme en production et en cherche le meilleur placement. Ce faisant, il tient évidemment compte de l'énergie d'heure creuse disponible et permet de voir l'influence sur l'intérêt du pompage, de facteurs tels que le chauffage par accumulation qui peut réduire l'énergie disponible dans certaines circonstances.

Ce programme, après dix minutes de passage à la CDC 6600 donne deux chiffres (entre autres) : le coût total des consommations, et le niveau de défaillance, tous deux en espérance mathématique annuelle.

Pour optimiser le parc, il faut déduire de ces essais des coûts marginaux, les comparer aux annuités d'investissements et de charges fixes des divers moyens de production, rectifier en conséquence la composition du parc, et progresser ainsi vers le parc optimum.

Le pompage, lui, est rebelle à cette méthode: il présente trop de paramètres et la définition de son service ne peut se réduire, on l'a vu, à l'énoncé de sa puissance disponible et d'un coût fixe de production. Il faut préciser le stock attaché à cette puissance, qui limite la fourniture certaines semaines, et calculer chaque semaine le coût spécifique de sa production.

C'est pourquoi, pour le pompage, on se résout à essayer un parc donné, défini par sa puissance et son stock accu- mulable par semaine (et on distingue sur la monotone le stock pompable dans les heures les plus creuses de la semaine et le stock pompable dans les heures un peu moins creuses des nuits 《ouvrables 》) et on optimise le reste du parc. Puis on essaye un autre parc de pompage.

Fort heureusement, on trouve, pour une large bande de pompage, qu'il n'y a que la quantité de thermique léger, type turbine à gaz (dit thermique $\mathrm{C}$ ), à ajuster pour rester à l'optimum. Ceci justifie la première comparaison grossière pompage-turbine à gaz. Il en serait différemment, sans doute, si dans le parc on introduisait un thermique $\mathrm{B}$ semiléger, à mi-chemin entre le $\mathrm{C}$, et le thermique $\mathrm{A}$ de $700 \mathrm{MW}$.

L'optimisation d'un parc avec pompage se fait donc comme illustré sur le graphique 5, et la valorisation attachée à un certain projet de pompage s'obtient par différenciation entre deux situations optimales, l'une comprenant ce projet, et l'autre non.

Résultats:

Vue d'ensemble des valorisations

Bien entendu, il s'agit de valorisations attachables à la fonction de transfert d'énergie d'heures creuses sur les heures pleines, le modèle n'analysant pas l'intérêt de la modulation à chaque instant de la fourniture. Ces valorisations 


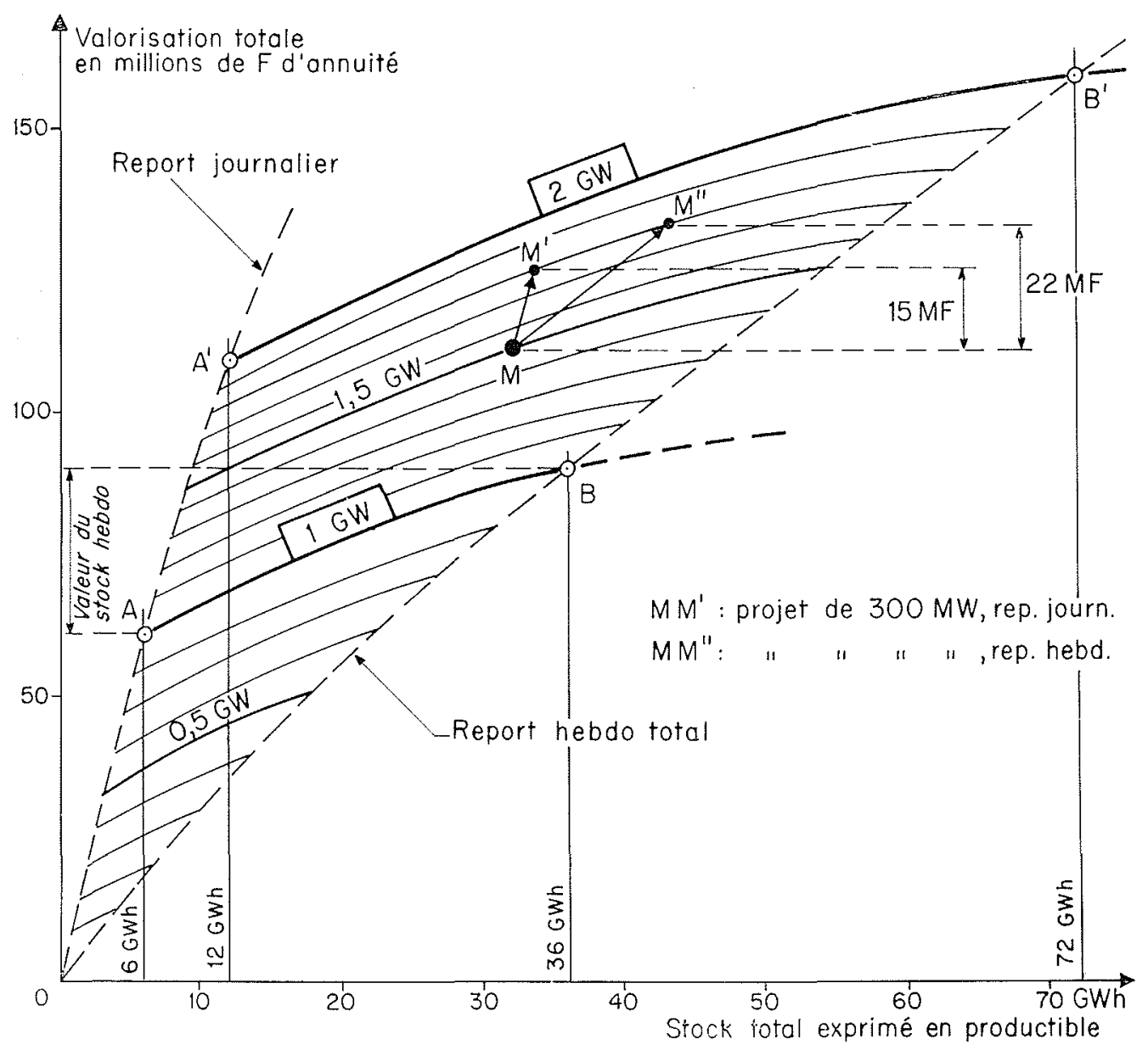

6/Représentation des valorisations du pompage.

qui résultent de la somme, cette année-là, des consommations épargnées, de la quantité de turbine à gaz économisée, et de la variation éventuelle de la défaillance chiffrée, s'expriment en millions de francs d'annuité de gain.

Une des différences qui apparaît avec le calcul grossier de l'approche initiale par comparaison directe avec la turbine à gaz, c'est que $1 \mathrm{GW}$ de pompage ne fait pas économiser $1 \mathrm{GW}$ de turbine à gaz, à cause de sa durée de fourniture, limitée par la capacité de ses bassins. Le rapport de substitution varie de presque 1 , s'il s'agit d'un premier GW de pompage (donc associé à beaucoup de turbines à gaz) de longue durée de fourniture, jusqu'à moins de 0,5 , s'il s'agit d'un GW de pompage s'ajoutant à un parc déjà important de pompage (donc associé à peu de turbines à gaz) et de courte durée de fourniture.

Pour représenter le tableau d'ensemble de ces annuités, on adopte la représentation des graphiques $7,8,9$ et 10 qui donnent pour les années 1980, 1985, 1988 et 1995, les valorisations globales en fonction des deux caractéristiques définissant, comme on l'a vu, un parc de pompage: la puissance et le stock qui y est associé.

Le graphique 6 explicite la signification de ce diagramme:

Le point A représente un parc de $1 \mathrm{GW}$ de report jourmlier: on lui associe un stock de $6 \mathrm{~h}$ de fourniture, soit $6 \mathrm{GWh}$ exprimés en productible (pour $8 \mathrm{~h}$ de pompage).
Le point $B$ représente un parc de $1 \mathrm{GW}$ utilisant au maximum les heures de pompage de fin de semaine.

En admettant $50 \mathrm{~h}$ de pompage possible du vendredi soir au lundi matin, on stocke $36 \mathrm{~h}$ de productible, soit $36 \mathrm{GWh}$ (et on peut, en repompant chaque nuit, produire $60 \mathrm{~h}$ dans la semaine à pleine puissance).

Entre $\mathrm{A}$ et $\mathrm{B}$, le parc est à report hebdomadaire partiel et en décrivant $A B$, on décrit, sur une courbe isopuissance, la variation de la valorisation attachée à la durée de la fourniture, de 30 à $60 \mathrm{~h}$ hebdomadaires.

La courbe pointillée gauche $\mathrm{AA}^{\prime}$ donne la succession des valorisations d'un empilage homogène de stations toutes journalières; la courbe pointillée droite $\mathrm{BB}^{\prime}$ les valorisations d'un empilage homogène de stations toutes hebdomadaires totales. A l'intérieur de ces courbes, on représente des états mixtes. Par exemple, le point $M$ représente un parc de 1,5 GW, en moyenne semi-hebdomadaire.

On comprend bien sur les graphiques $7,8,9$ et 10 que les valorisations de pompage ne soient pas simples à présenter, car elles dépendent :

- de l'année considérée;

- du type de pompage considéré;

- de la quantité de pompage déjà installé;

- de la nature du pompage déjà installé.

Il faut donc, pour qu'une valorisation ait un sens, l'énon- 


\section{P. GERARD}
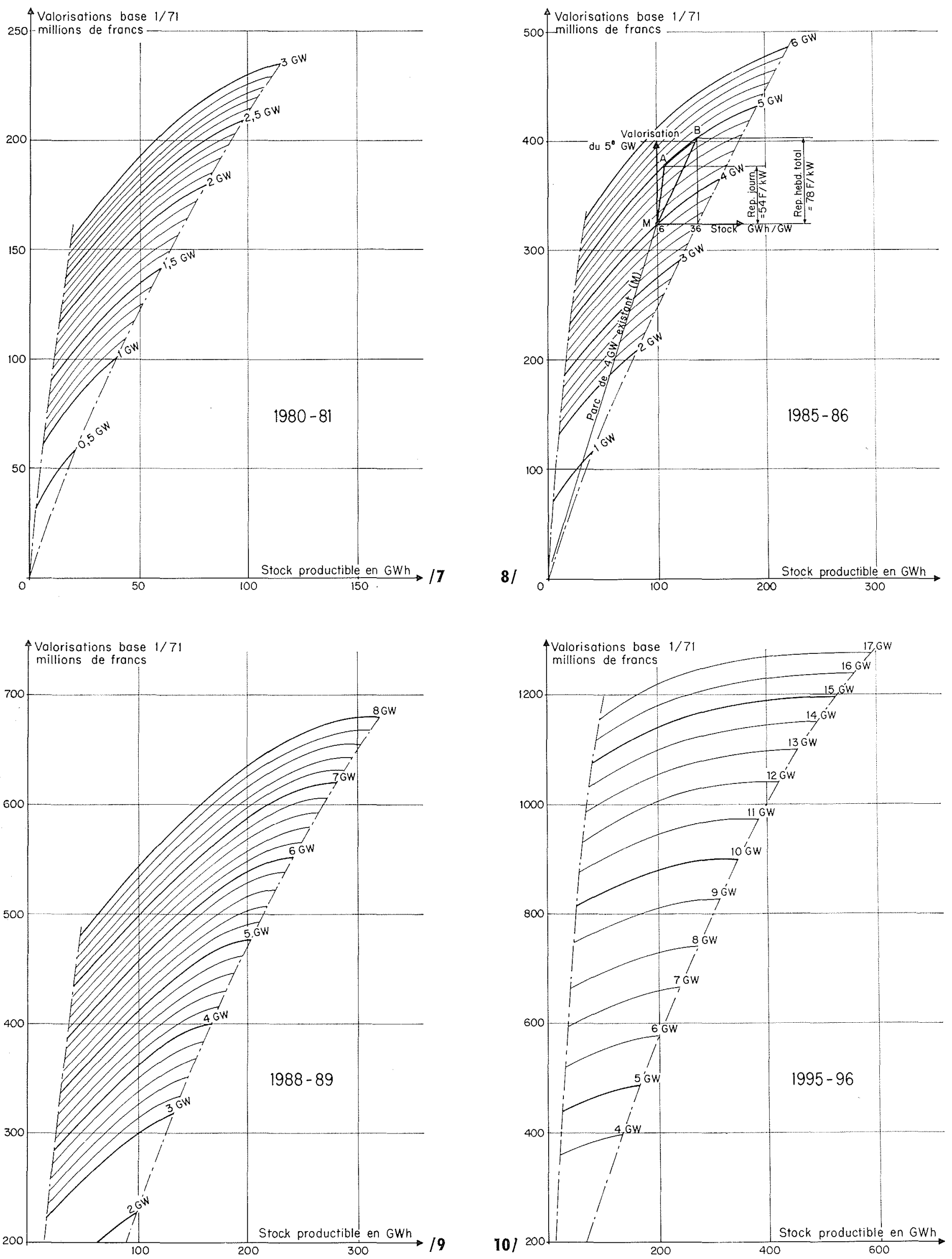
cer ainsi : par exemple: «valorisation du $5^{\circ} \mathrm{GW}$ en 1988 1989, les 4 GW précédents étant composés de telle manière, et ce $5^{\circ} \mathrm{GW}$ étant de tel type $\gg$.

Tout ceci implique qu'il s'agit de parcs optimisés : s'il y a peu de pompage, il y a plus de turbine à gaz et vice-versa.

Les idées générales qui se dégagent de ces valorisations sont les suivantes:

- on voit clairement la décroissance de la valorisation des GW au fur et à mesure qu'on les empile dans une année donnée, quelle que soit leur nature, décroissance qui traduit la saturation des besoins et la réduction progressive de l'énergie d'heure creuse disponible;

- on voit le supplément de valorisation des GW à report hebdomadaire par rapport aux GW à report journalier, ce supplément tendant à se réduire, bien entendu, quand le parc se sature en report hebdomadaire possible, gros consommateur d'énergie d'heures creuses;

- au cours des ans, on voit la dilatation générale des valorisations;

- on voit que la valeur intrinsèque du report hebdomadaire, très grande jusqu'en 1985, décroît, puis devient faible en 1995

Notons que toutes ces idées générales sont applicables au transfert d'énergie, qu'il soit par pompage hydraulique ou par installation pneumatique. Dans ce dernier cas, il suffit de considérer qu'on a un ensemble composé d'une turbine à gaz classique et d'un système de transfert d'énergie.

\section{Application au dimensionnement des installations}

La pente des courbes isopuissances donne la valeur attachable au stock, c'est-à-dire au kWh stocké. En analysant ces courbes, et en se plaçant dans une stratégie de développement probable (le raisonnement est, en fait, itératif), on dégage que le $\mathrm{kWh}$ stocké pour faire du report hebdomadaire (c'est-à-dire la part des bassins employée une fois par semaine) est gratifié d'une rente annuelle de $0,8 \mathrm{~F}$ en 1980 ; $1,0 \mathrm{~F}$ en $1985 ; 0,7 \mathrm{~F}$ en 1988 et $0,35 \mathrm{~F}$ en 1955 . En actualisant tout ceci, on voit qu'on peut dépenser 6 à $7 \mathrm{~F}$ pour stocker un $\mathrm{kWh}$ pour un bassin mis en service en 1980 , 4 à $5 \mathrm{~F}$ pour une mise en service en 1988 .

Si les bassins coûtent plus de 7 à $8 \mathrm{~F} / \mathrm{kWh}$, il faut les employer à du report journalier, c'est-à-dire associer à ces bassins une plus grande puissance. En revanche, le projet sera probablement repoussé plus tard dans l'échéancier des réalisations, car on voit que ce type d'installation n'a qu'une place limitée jusqu'en 1985, du fait de la valorisation sensiblement moindre qui lui est accordable.

Si le bassin supérieur coûte moins de $0,5 \mathrm{~F} / \mathrm{kWh}$ stocké, c'est-à-dire dix fois moins cher que le coût convenable pour du stockage hebdomadaire, il est logique d'employer ce bassin pour du report saisonnier. Le coût du bassin inférieur déterminera alors si on ajoute ce report saisonnier seulement à du report journalier, ou bien aussi à du report hebdomadaire (schéma 3).

Ce coût de $6 \mathrm{~F} / \mathrm{kWh}$ pour le stock hebdomadaire, qui conduit à $30 \mathrm{~F} / \mathrm{kWh}$ pour le journalier qui sert cinq fois plus souvent, est bien homogène avec les chiffres donnés dans l'approche simplifiée initiale.
Esquisse

d'un échéancier

On compare le panorama des valorisations ainsi définies avec le coût des projets de pompage, ceux-ci étant préalablement optinisés conformément à leur vocation comme on vient de le dire. En fait, il y a un va-et-vient entre l'échéancier et cette optimisation, puisqu'une l'une est liée à l'autre.

Le coût du projet comprend les investissements avec les frais d'étude et d'argent, les frais fixes d'amortissement et de conduite, et la charge de transport d'énergie, fonction de la localisation du projet sur le réseau. Le total divisé par le taux d'actualisation donne l'annuité de coût à comparer avec la valorisation.

Par exemple : $4 \mathrm{GW}$ de pompage étant déjà placés pour le parc en 1985 , on cherche si un 5 ime GW est plaçable et de quel type il doit être (graphique 8). Pour ce $5^{\text {tme }} \mathrm{GW}$, on trace la courbe de la rente annuelle fonction du stock qui y est attaché. On compare avec les annuités de coût des sites en portefeuille, et pour tous ces sites, on actualise jusqu'à l'infini l'enrichissement qu'ils apporteront.

On n'est certainement pas loin de la stratégie de développement optimale en se fixant la règle de choix suivante: le site retenu pour être mis en service en 1985, au titre du 5ime GW, doit :

a) être déjà renté au taux obtenu (10\% actuellement) en 1985 ;

b) avoir la meilleure rentabilité actualisée de 1985 à l'infini parmi tous les sites en portefeuille.

Ce site une fois trouvé et placé, on opère de même pour rechercher le placement d'un éventuel $6^{\text {ime }} \mathrm{GW}$.

Quand on ne trouve plus de site renté en 1985, le parc est saturé, et pour placer de nouveaux projets, il faut attendre quelques années pour que les valorisations soient plus grandes. A ce parc maximal de pompage, correspond une certaine quantité optimale résiduelle de turbines à gaz, toujours présentes.

En confirmation de ce qui a été dit plus haut, on trouve que le total des besoins de pointe est fonction de la variance de la principale donnée aléatoire: la demande. Suivant qu'on adopte un écart-type de 5 ou de $2 \%$, l'ordre de grandeur des valeurs à prévoir est donné par le tableau ci-dessous :

\begin{tabular}{|c|c|c|c|c|}
\hline \multirow[b]{2}{*}{ ANNÉES } & \multicolumn{2}{|c|}{$\begin{array}{l}\text { ECART-TYPE } 5 \% \\
\text { (pour mémoire) }\end{array}$} & \multicolumn{2}{|c|}{ ECART-TYPE $2 \%$} \\
\hline & Pompage & $\begin{array}{l}\text { Turbine } \\
\text { à gaz }\end{array}$ & Pompage & $\begin{array}{c}\text { Turbine } \\
\grave{a} \text { gaz }\end{array}$ \\
\hline $1980-1981$ & $2,3 \mathrm{GW}$ & $3 \mathrm{GW}$ & $2,5 \mathrm{GW}$ & $1,2 \mathrm{GW}$ \\
\hline $1985-1986$ & $5,0 \mathrm{GW}$ & $5 \mathrm{GW}$ & $5,5 \mathrm{GW}$ & $2,3 \mathrm{GW}$ \\
\hline 1988-1989 & $7,5 \mathrm{GW}$ & $7 \mathrm{GW}$ & $8,0 \mathrm{GW}$ & $3,5 \mathrm{GW}$ \\
\hline 1995-1996 & $15,0 \mathrm{GW}$ & $14 \mathrm{GW}$ & $16,0 \mathrm{GW}$ & $7,0 \mathrm{GW}$ \\
\hline
\end{tabular}

On voit que la quantité optimale de transfert d'énergie par pompage, résultant essentiellement des données de l'année de prévision moyenne est peu sensible à la variance de la demande, tandis que la quantité de turbine à gaz, y est très sensible. 


\section{P. GERARD}

Le programme de pompage de transfert d'énergie qu'on peut prévoir peut se résumer ainsi :

En ce qui concerne le volume:

- de 1975 à 1980, on doit mettre en service de l'ordre de $0,5 \mathrm{GW}$ par an;

- de 1980 à 1985, on doit mettre en service de l'ordre de $0.75 \mathrm{GW}$ par an;

- de 1985 à 1990, on doit mettre en service de l'ordre de 1 GW par an;

- le volume optimal de turbines à gaz est de l'ordre de $50 \%$ du volume de pompage.

En ce qui concerne la nature :

- jusqu'en 1985, on doit mettre en service surtout du pompage à grand stock (report hebdomadaire ou éventuellement saisonnier);
- de 1985 à 1990, progressivement du pompage à grand stock et du pompage journalier;

- au-delà de 1990, probablement presque uniquement du pompage journalier.

Cela correspond assez bien au portefeuille de sites déjà prospectés. Bien entendu, quand il s'agit de prospective lointaine (au-delà de 1985 ou 1990), ces résultats doivent être annoncés avec prudence, car ils dépendent de ce que sera la structure de la demande, compte tenu du développement plus ou moins important que prévu du chauffage à accumulation, de la voiture électrique, de l'évolution géographique de la demande et des charges de transport qui en résultent, des progrès techniques dont bénéficiera telle ou telle technique, etc

Ces facteurs nuanceront les conclusions actuelles; il est cependant peu probable qu'ils les bouleversent.

Discussion

Président: M. H. DE Maublanc

M. le Président félicite M. P. GÉrard de son brillant exposé sur l'économétrie du pompage et souligne l'importante part qui lui revient dans la mise au point des méthodes qu'il a exposées.

11 ouvre ensuite la discussion par les commentaires ci-après:

Pour ma part, je retiens de l'exposé de M. GÉRARD que, finalement, lHydraulique trouvera dans le pompage un deuxième souffle. Les chiffres évoqués par M. GÉrard sont d'un ordre de grandeur tel que, compte tenu de la puissance hydraulique installée dans notre propre pays (de l'ordre de $16 \mathrm{GW}$ ) et des développements envisagés pour le pompage $(0,5$ à $1 \mathrm{GW}$ par an), on peut penser que les stations d'accumulation par pompage représenteront dans un avenir assez proche 10 à $12 \%$ de la puissance totale des usines françaises.

Comme la puissance installée ne double pas tous les dix ans (mais peut être tous les douze ou treize ans du fait que l'utilisation s'améliore), la production doublant à peu près tous les dix ans, le pare des stations de pompage représentera un outil de production assez impressionnant.

Fort heureusement, d'ailleurs, les projeteurs de nos différentes Régions d'équipement hydrauliques, et le service de M. RACT-Madoux ont déjà inventorié quelques dizaines de sites convenant à des stations de pompage dans notre pays, une bonne quinzaine paraissent, dès à présent, sisceptibles d'être retenus eu égard à Jeur situation topographique et géographique par rapport au réseau. Nous avons là de belles perspectives et je pense que nos ingénieurs, nos clients bien sûr et aussi nos constructeurs s'en réjouiront.

M. Dumarne (E.D.F.-D.E.P.H.) intervient en ces termes:

Je voudrais faire quelques remarques en complément à ce que vient de dire M. Gérard concernant lapplication de la « Note Bleuc 》 à la constitution des programmes d'Hydraulique classique.

$\mathrm{Si}$, en 1953, au moment de l'établissement de la première « Note Bleue » on s'était posé le problème de l'optimisation du pare hydraulique pour les vingt-cinq années à venir, on aurait buté sur la même difficulté que celle qui vient d'être exposée à propos du pompage : la valeur des parcs successifs n'aurait pu être fixée intrinsèquement mais aurait dépendu de l'ordre choisi pour les réalisations successives.

Aussi bien s'est-on contenté de construire une méthode marginale qui ne pouvait donc s'appliquer qu'à un ensemble restreint et sitôt que la structure du parc avait changé suffisamment, on refondait la "Note Bleue », d'où les révisions successives de cette méthode tous les deux ou trois ans.

Mais, pour chaque période où une série de règles était applicable, on pouvait effectivement fabriquer un programme marginal-optimal en se fixant en outre une contrainte supplémentaire: le volume d'investissements financiers annuels, fonction des possibilités budgétaires de l'Etablissement.

On peut ne pas le faire pour le pompage parce que, dans le choix entre ce moyen et les autres, la part investissements difière beaucoup moins que dans le cas de l'hydraulique classique et des moyens concurrents.
On pourra objecter qu'avec la règle d'ajustement découlant de la « Note Bleue » on n'a peut-être pas choisi le cheminement optimal pour réaliser les équipements. N'importe, on peut penser qu'on ne s'en est point trop écarté car, à l'inverse du pompage, nul problème de fourniture d'énergie d'heures creuses pour pomper ne vient compliquer le problème.

Et d'ailleurs la structure du parc ainsi réalisée rend les services qu'on en attend: c'est la meilleure justification de nos choix antérieurs.

M. Gérard partage le point de vue de M. Dumaine mais souligne le fait que les conclusions tirées de la « Note Bleue» sont fortement infuencées par le laux d'actualisation admis; or, celui-ci a varié assez largement dans le passé.

Il faut dire, observe $M$. le Président, que pratiquement, le taux d'actualisation est imposé, non sans une discussion préalable, le concessionnaire entendu. 11 peut varier entre 8 et $12 \%$ suivant la nature des investissements.

En fait, la plupart, sinon la totalité, de nos calculs économiques de production et de distribution sont faits avec le taux de $10 \%$ Peut-être, un jour, si l'argent se fait beaucoup plus abondant, si les priorités deviennent différentes de ce qu'elles sont, pourra-t-on modiffer ce taux et l'abaisser un peu; mais je considère que c'est très peu probable, les taux iront plutôt dans le sens de l'augmentation.

M. Lefoulon expose, comme suit, lintérêt de faire contribuer en basses eaux les usines hydroélectriques au fil de l'eau à la production d'énergie de pointe.

Il y a quelques années, on envisageat les centrales de pompage pour la production de la puissance de pointe d'une utilisation journalière de 3 à 4 heures (au maximum 10 heures). L'exposé de M. GÉrard montre qu'actuellement les centrales de pompage, en particulier Montezic, sont conçues pour produire, non seulement l'hiver mais toute l'année, de l'énergie «d'heures pleines » correspondant à une utilisation d'au moins 2000 heures.

Bien que l'importance relative de la pointe ait tendance à diminuer avec l'abaissement des durées de travail et l'aménagement des horaires, il reste à produire une puissance d'une utilisation de 50 à 300 heures par an. Qui la fournira? M. GÉRARD en laisse la production aux turbines à gaz qui ne viendraient pas ainsi en concurrence avec les usines de pompage hydrauliques.

Je suis d'accord sur l'utilisation des turbines à gaz qui nécessitent le minimum d'investissement et dont la technique évolue rapidement. Toutefois, je me permets de rappeler que depuis longtemps, je suggère que la puissance de pointe, aussi paradoxal que cela puisse paraître, doit être produite par une puissance installée qui ne coûte aucun investissement et j'entends par là lexploitation des usines au fil de l'eau « en éclusées », en hiver, lorsque l'hydraulicité est moyenne ou faible. A cette époque, les usines ont de la puissance disponible et peuvent très bien moduler une plus ou moins grande partie du 
débit de 24 heures pour produire de la puissance de pointe, 2 heures le matin et 2 ou 3 heures le soir. Les usines de basse chute en cascade de l'Isère, de la Durance, de la Garonne, du Rhône et du Rhin et d'autres peuvent produire gratuitement plusieurs centaines de mégawatts de pointe (nonobstant les lâchures des réservoirs et usines en tête de vallée qui ne font, à l'aval, qu'augmenter le débit moyen journalier que l'on peut remoduler).

Je pense que si on n'a pas utilisé cette exploitation «en éclusées» des usines au fil de l'eau, c'est qu'on n'en a pas éprouvé le besoin. Je persiste à croire qu'il serait anti-économique de ne pas le faire dans lavenir lorsque la production nucléaire fournira de plus en plus de l'énergie de 24 heures en demandant un petit effort au dispatching et à la production hydraulique.

Jajoute que, compte tenu de l'importance des investissements, les aménagements hydrauliques de pompage comme les aménagements hydroélectriques de lac saisonnier ne sont économiquement viables, que s'ils produisent de l'énergie avec une utilisation de la puissance installée d'au moins 1800 à 2000 heures, c'est-à-dire de l'énergie d'heures pleines et non de l'énergie de pointe comme on l'a souvent dit improprement.

M. GÉRARD signale qu'un groupe de travail a été constitué, au sein d'Electricité de France, pour étudier la quantité d'énergie de pointe susceptible d'être obtenue par une modulation appropriée de la production d'étiage des usines de basses chutes. L'étude a porté sur quarante-huit usines, confirme M. Dumaine, mais eu égard aux nombreuses contraintes imposées par ce mode d'exploitation, son intérêt a été jugé assez faible.

M. Paris (E.N.E.L.-C.N.S.P., Rome) intervient ensuite en ces termes:

J'ai suivi avec intérêt le rapport de $M$. GÉRARD que je considère d'une importance fondamentale pour la détermination de l'évolution du parc des aménagements de pompage dans un système électrique, et il est sans doute un des rapports les plus complets et approfondis que j'ai eu la possibilité de connaître.

Les questions que je voudrais bien poser sont très nombreuses, mais, en raison du manque de temps, je poserai une question seulement qui peut avoir une importance fondamentale sur les résultats, bien qu'elle puisse sembler de caractère marginal

M. GÉraRd, en effet, chiffre l'ordre de grandeur de la valeur économique du kWh non fourni à quelque $3 \mathrm{~F}$, valeur qui peut, peut-être, être quelque peu réduite dans le cas des défaillances moins graves. II me semble que cette valeur-là est particulièrement réduite: dans divers pays, on admet pour le $\mathrm{kWh}$ non fourni, un prix bien plus élevé - soit explicitement, soit implicitement - dans la programmation des systèmes électriques de production. L'adoption d'une valeur plus élevée pour le kilowatt/heure « de défaillance » implique l'exploitation d'un nombre plus grand d'usines à utilisation très faible, et corrélativement la modification du parc des aménagements de pointe qui sont nécessaires, du rapport entre turbine à gaz et pompage et enfin des capacités des réservoirs des usines de pompages elles-mêmes.

Il semble intéressant de connaître les critères qui ont abouti à la valeur choisie, et de savoir si on a essayé de modifier cette valeur afn de se rendre compte de la modification des conclusions qui en résulte.

Il serait intéressant aussi de savoir si, pendant les périodes de défaillance, la puissance disponible est utilisée totalement, ou si l'on garde une partie de celle-ci en fonctionnement comme réserve tourinante.

Dans notre étude, répond M. GÉrarD, trois niveaux de défaillance ont été considérés: une défaillance d'une profondeur de $1,4 \mathrm{GW}$ est taxée à raison de $0,50 \mathrm{~F}$ par $\mathrm{kWh}$; une défaillance comprise entre 1,4 et $4 \mathrm{GW}$ est taxée à $1,50 \mathrm{~F}$ par $\mathrm{kWh}$; et au-delà de $4 \mathrm{GW}$, elle esi taxée $3 \mathrm{~F}$ par $\mathrm{kWh}$.

Il en résulte un coût moyen de défaillance poư les parcs optimisés, qui est de l'ordre de $1,3 \mathrm{~F}$ par $\mathrm{kWh}$. Ce coût est homogène avec le coût du kilowatt/heure qui serait produit par une turbine à gaz marginale qui fournirait en moyenne 50 heures par an, espérance mathématique de la durée de défaillance à laquelle on aboutit.

M. GÉrard précise que, dans ses calculs, en cas de défaillance, on admet que les « réserves tournantes » restent disponibles à leur niveau normal

M. ANDRé demande comment se place, au point de vue rentabilité, la solution consistant à augmenter la production d'énergie de pointe par suréquipement d'usines hydroélectriques existantes.

Assez mal, répond M. GÉRARd, car ces suréquipements sont généralement plus coûteux qu'on ne le pense et les travaux entrepris sur des usines existantes peuvent présenter bien des aléas. D'autre part actuellement, et semble-t-il pendant la prochaine décennic, ces suréquipements ne correspondent guère aux besoins car on peut les assimiler au point de vue qui nous occupe à des usines sans réservoir or, nous manquons davantage d'énergie en réserve que de puissance installée.

$M$ le Président clôt la discussion en remerciant tous ceux qui on contribué à l'animer. Il donne ensuite la parole à M. Ponsy pour l'exposé de sa communication. 


\section{Abstract \\ Economic assessment \\ of pumped storage schemes by mathematical simulation of plant operation. Applications to site selection and development}

For the last several years Electricite de France have been assessing the economics of hydroelectric projects by comparing the project with a fictitious themal power station which could be built on the same site and which would give equivalent service.

Computer programmes now available can be used to determine the best overall combination of the various power production schemes which would satisfy a given demand at minimum total cost. In the work presented here a mathematical model is used to simulate operation of the entire French network with a view to finding the optimal amount and type of pumped storage which should be installed.

Before giving the results of this overall model study, pumped storage and gas turbines are compared and several important general ideas are deduced from this comparison.

Based on French prices, Figure 1 gives the comparative cost of punping stations with daily (Revin) and weekly time cycles (Montézic). Excluding the storage structures, this cost is in the order of $300-400 \mathrm{~F}$. The difference is due to storage costs. This is expensive for the first type $(30 \mathrm{~F} / \mathrm{kWh})$ but much less so for the second $(2 \mathrm{~F} / \mathrm{kWh})$. With a day cycle-time storage only occurs over 6 hours, the cost being $180 \mathrm{~F} / \mathrm{kW}$. For the second case, the cost is only $72 \mathrm{~F} / \mathrm{kW}$ with a 36 -hour basin (weekly cycle which provides 60 hours per week at full capacity).

However, inexpensive storage is only available in mountainous areas (high falls) and transport costs are high $(200 \mathrm{~F} / \mathrm{kW})$ to the industrial power-consuming regions situated in the main well north of the mountains.

By and large, the six-hour supply delivered to the Paris area by a day-type scheme gives an inchusive cost of $650 \mathrm{~F} / \mathrm{kW}$ whereas the twelve-hour supply from a weekly scheme amounts to approximately $750 \mathrm{~F} / \mathrm{kW}$, transport included.

We are now in a position to compare pumped storage with gas turbines and this is done in Figure 2 which shows (a) that the short supply time (4 or 5 hours) is undoubtedly the economical domain for pumping (white area) unless a large number of cycles (over a 100) are required each year, in which case the economics are in favour of the longer duration and (b) that the economical domain for the gas turbine (shaded area) corresponds to the longer duration supply, this being the case, however, only on a few occasions in any given year.

Pumped storage and gas turbines are thus shown to be complementary.

Seasonal time-cycle pumping stations have also been considered (see Figure 3). These are of interest in that they are able to modulate the duration of their supply from one week to the next. However, scasonal stations do not solve the problem of variation from year to year: this remains the special field of the gas turbine.
Figure 4 shows that, although on average the gas turbine is only used a few hours a year (Figure $4 \mathrm{c}$ ) and is the uppermost plant type on the load duration curve, a plot of this kind in fact expresses a mathematical expectancy which is the sum of a large number of weeks in normal years with no recourse to gas turbines (Figure $4 \mathrm{~b}$ ) and abnormal weeks in difficult years during which the gas turbine is used for a long supply duration (Figure 4 a) according to the ideas given above.

In order to calculate the best breakdown between pumping and gas turbines, it is therefore necessary to use mathematical models which simulate the entire network (thermal and hydraulic) on the basis of probabilities, i.e. demand probability, probability of availability of thermal and hydroelectric power and energy availability probability during slack hours.

Figure 5 shows the result of such a test. Here the amount of peak-hour plant is optimized by adjusting the number of gas turbines ( $\mathrm{x}$-axis) to achieve minimal total cost (y-axis), this being the sum of annual gas-turbine cost (curve A), power consumption (B) and extent of power default (C).

The "value" of a pumping scheme is given by the difference between the total cost obtained for the optimized network in which the scheme appears and the network cost without the scheme.

Figure 6 shows how this "value" ( $y$-exis) varies with the reserve associated with each GW of pump power ( $x$-axis). The y-value of $A$ is the value of $1 \mathrm{GW}$ for the daily cycle where as the $y$-value of $B$ is for $1 \mathrm{GW}$ of the weekly cycle. Dashed letters A' and B' are for $2 \mathrm{GW}$.

MM' represents a $300-\mathrm{MW}$ daily pumping scheme which has been added, during the year in question, to the pump plant defined by point $\mathrm{M}$; its value is $15 \times 10^{\mathrm{h}} \mathrm{F}$. MM" represents a $300-\mathrm{MW}$ weekly scheme, the value of which is $22 \times 10^{6} \mathrm{~F}$.

Shown on the same type of plot in Figures 7 to 10 is the overall pumping "value" inserted into the French network during years $1980,1985,1988$ and 1995.

By tracing on these plots the best pumped-storage development strategy for France, it is found that quantities and types to be installed are as follows:

(i) Quantities : $0.5 \mathrm{GW}$ per year between 1975 and 1980, $0.75 \mathrm{GW}$ per year between 1980 and 1985, approximately $1 \mathrm{GW}$ per year after 1985 and about half of all the preceding power in the form of gas turbines;

(ii) Type of pumping : mainly the weekly type up to 1985 , changing gradually to a predominance of the daily type thereafter. 